\title{
Modeling of integrated extended cavity InP/InGaAsP semiconductor modelocked ring lasers
}

\author{
Erwin A. J. M. Bente • Yohan Barbarin • \\ Martijn J. R. Heck • Meint K. Smit
}

Received: 29 June 2007 / Accepted: 21 January 2008 / Published online: 8 February 2008

(C) The Author(s) 2008

\begin{abstract}
In this paper a model and simulation results of integrated semiconductor passively modelocked ring lasers are presented. The model includes nonlinear effects such as two-photon absorption and a non-linear refractive index, a logarithmic gain-carrier relation, and concentration dependent radiative and non-radiative carrier recombination rates. The optical bandwidth of the system is controlled by a digital filter. The model has been used to simulate two geometries of ring modelocked lasers. The first is a symmetric design, where the two counter propagating pulses in the cavity experience the same amplification and absorption. The second is an asymmetric design where the differences for the two directions of pulse propagation are maximised. Simulation results show that a symmetrical cavity shows a several times wider window for its operating parameters for stable modelocking.
\end{abstract}

Keywords Integrated optics · Mode-locked lasers · Modelling - Optical pulse generation Semiconductor lasers $\cdot$ Semiconductor optical amplifiers(SOA)

\section{Introduction}

Semiconductor modelocked lasers (MLLs) at $1.55 \mu \mathrm{m}$ can be useful devices for a number of applications (Williams et al. 2004). In particular for high speed optical communication systems they can be used as pulse sources in OTDM systems (Takara 2001), multiwavelength sources in DWDM systems or all optical clock recovery (Ohno et al. 2004). Other applications can be e.g. in optical fibre based sensor systems. Passively modelocked devices are of interest due to the fact that they require relatively low cost DC electrical supplies to operate. In such devices typically a short section of the active waveguide is reversely biased to form a saturable

E. A. J. M. Bente $(\varangle) \cdot$ Y. Barbarin · M. J. R. Heck · M. K. Smit COBRA Research Institute, Eindhoven University of Technology, Eindhoven $5600 \mathrm{MB}$, The Netherlands e-mail: e.a.j.m.bente@tue.nl

Y. Barbarin

ETH Zürich, Wolfgang-Pauli Strasse 16, 8093 Zurich, Switzerland

e-mail: barbarin@phys.ethz.ch 
absorber (SA) (Derickson et al. 1992). An important advantage of semiconductor devices is not so much that they can be more robust and smaller than solid-state laser sources, but that such lasers can be more easily mass-produced and integrated on a single chip with other optical devices such as optical switches, filters and amplifiers using active-passive integration technology. At the COBRA Research Institute we are pursuing the development of such integrated optical circuits. The ring laser geometry for the semiconductor MLL is of particular interest for two reasons. The first reason is that is can be easily located in the middle of a chip somewhere without the need to fabricate etched mirrors. Secondly, a modelocked ring laser, as also will follow from the work presented here, will typically operate in a colliding pulse modelocking mode. This will provide more stable modelocking and a more deeply saturated absorber. Active-passive integration however also brings additional design freedoms. One can design ring cavities where the total length of the cavity, the amplifier and the absorber can be set more independently than in an all active device since part of the cavity can be a passive waveguide. Also the relative position of the amplifier, absorber and output coupler can be varied.

In this paper we present a model to simulate semiconductor MLLs that can be realised with active-passive integration technology to get to an understanding of the mechanism of passive modelocking and to be able to design advanced devices. In particular we investigate the possibility of influencing the modelocking stability and the balance of optical power in the two directions by the positioning of the amplifier, absorber and output coupler. The model that has been developed and which is presented here is a bi-directional time domain model for ring modelocked lasers (RMLLs). The modelocking mechanism studied is a passive modelocking using a slow SA. The SA is a short semiconductor optical amplifier (SOA) section that is reversely biased. The model is applied to the bulk $1.55 \mu \mathrm{m}$ InGaAsP active medium, which is used in the active-passive integration scheme presented by Barbarin et al. (2005a). The model may also be used, with the appropriate parameter values, with quantum well gain media. The passive components are passive waveguides and passive couplers and the active components which all have an SOA layer structure and which can form an amplifier, a SA, or an isolation section between amplifier and SA.

The first reported work on the modelling of modelocking using a slow SA is from Haus (1975). Then the research in this field has grown with the development of semiconductor MLLs in the 90s. As was identified and discussed by Avrutin et al. (2000), there are two main types of time-domain modelocking theories. There are the lumped-element models (Koumans and van Roijen 1996; Leegwater 1996) and the fully distributed time-domain models (Yu et al. 1998). Another approach that is well developed is a travelling wave model (Zhang and Carroll 1992; Radziunas 2006; Bandelow et al. 2006). Recently Heck et al. (2006) presented a model with a lumped-element approach where the propagation of the pulse through the SOA and the SA were calculated using a distributed time-domain model. The SOA rate equations used in his model are based on a model from Tang and Shore (1999) which includes carrier heating $(\mathrm{CH})$ effects, two photon absorption (TPA) and ultrafast nonlinear refraction (UNR). These non-linear effects are included in the same way in the model presented in this paper. The model is fully in the time domain except for the bandwidth limitation in the laser. It is bi-directional to describe realistic laser systems which usually operate with pulses travelling in both directions. For the description of the SOA, the model of Tang and Shore has been extended with bidirectional fields, separate radiative and non-radiative carrier recombination processes (Olshansky et al. 1984) and a logarithm gain-carrier relation. This gain relation has been validated for the bulk material used in the RMLL described in Barbarin et al. (2006). The effect of the limited optical bandwidth of the SOA is not included in the rate equations of the SOA, but in a separate element in the model. This element is a 
bi-directional digital low-pass filter which stabilizes the system by limiting the bandwidth each roundtrip, in accordance with the spectral gain profile of the SOA/SA combination.

The model and study presented in this paper is similar to the model and studies presented by $\mathrm{Yu}$ et al. (1998). In that work the effects of the relative position of the amplifier and absorber sections, as well as multiple absorber sections are investigated in all-active ring lasers using a travelling wave model. The main differences with the model and work here are that we have included non-linear effects in the amplifier, applied our model to extended cavity lasers with passive sections that are several times longer than the amplifier and we have used a digital filter for bandwidth control. The simulation results and operating modes found are discussed in detail. Avrutin et al. (1999) have also reported on the simulation and experiments concerning modelocked semiconductor ring laser configurations. They have described ring lasers with multiple absorbers in order to obtain higher modelocking frequencies through harmonic modelocking. The simulations presented there were however based on a frequency domain model using cold cavity modes.

The remainder of this paper consists of two main sections. In the following section, the model for RMLL is described in detail. In Sect. 3 the model is used for the simulation of symmetric and asymmetric $20 \mathrm{GHz}$ modelocked ring lasers and the results are discussed.

\section{The integrated MLL model}

A bi-directional time domain model of a modelocked ring laser was developed that is based on a set of rate equations for a SOA. This system of differential equations relates the density of photons and charge carriers in the device to the injection current. Five different components are used in the extended cavity RMLL: passive waveguides, amplifiers, absorbers, isolation sections and a coupler. In this model, the ring laser is divided into segments that we assume equal in optical length (Fig. 1). There are five types of segment required for the different components. Uniform photon and carrier densities (for active components) are assumed in each segment. The time for the light to travel through one segment is $25 \mathrm{fs}$ which is the discretization time $\left(T_{\text {seg }}\right)$. Each $25 \mathrm{fs}$ the evolution of the photon densities (clock wise $(\mathrm{CW})$ and counter clock wise (CCW)) and carrier densities is calculated for all segments. Then the photon densities are transferred to the neighbouring segments and the carrier density values are kept in the same active segments for the next step.

The SOA rate equations of the model presented here include the nonlinear effects $\mathrm{CH}$, TPA and UNR in the same formalism as Tang and Shore (1999). The rate equations have been extended to take into account bidirectional fields (CW and CCW). Also, the model presented here makes the distinction between radiative and non-radiative carrier recombination processes (Heck et al. 2006). Finally, in the rate equations of the SOA a logarithmic gain model is used that has been validated experimentally by us (Barbarin et al. 2006). The gain-carrier relation used is given in Eq. 1, where $a N$ is the linear gain coefficient, $N_{0}$ the material transparency carrier density and $N$ the carrier density. The relation is illustrated in Fig. 2. The average carrier density in the amplifier inside the laser above threshold is around two times $N_{0}$ in the systems modelled. A linear gain-carrier relation is commonly used in lasers since the typically the variation in carrier density in the amplifier in time is over a limited range. However it can be seen that the slope of the curve becomes smaller at higher values of $\mathrm{N}$ and that the linear gain coefficient would need to be corrected (Koumans and van Roijen 1996). Using the logarithmic relation, no correction is needed. Furthermore, this relation is more flexible for the simulation of various type of RMLL with different amplifier length and thus different averaged carrier densities. 


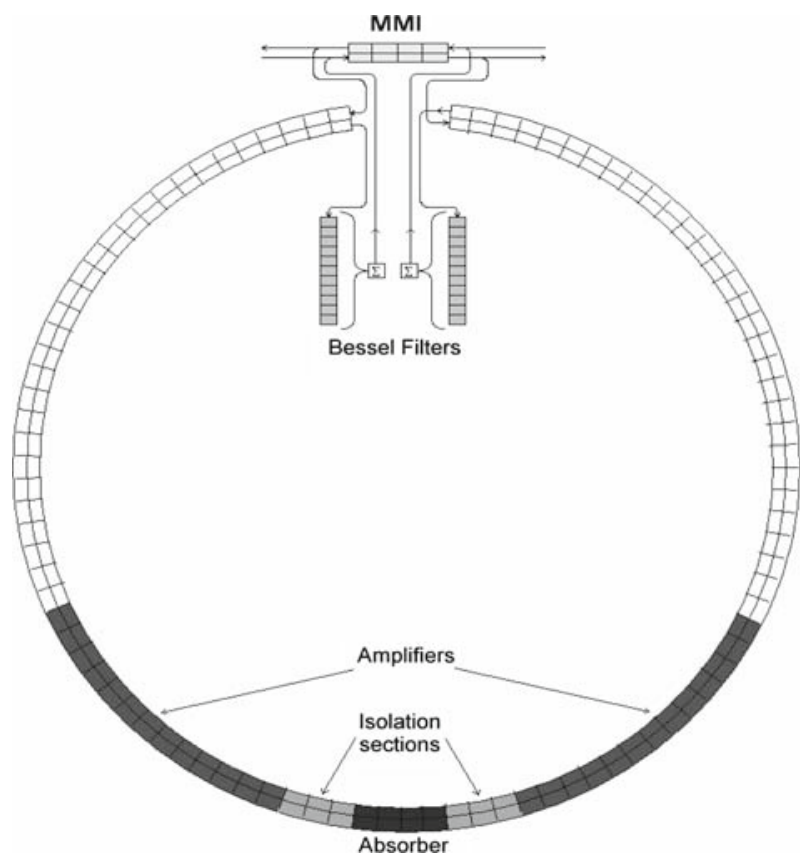

Fig. 1 Diagram of the symmetric ring modelocked laser segmented for the simulation. The connections between the coupler, the ring and the Bessel filters are shown

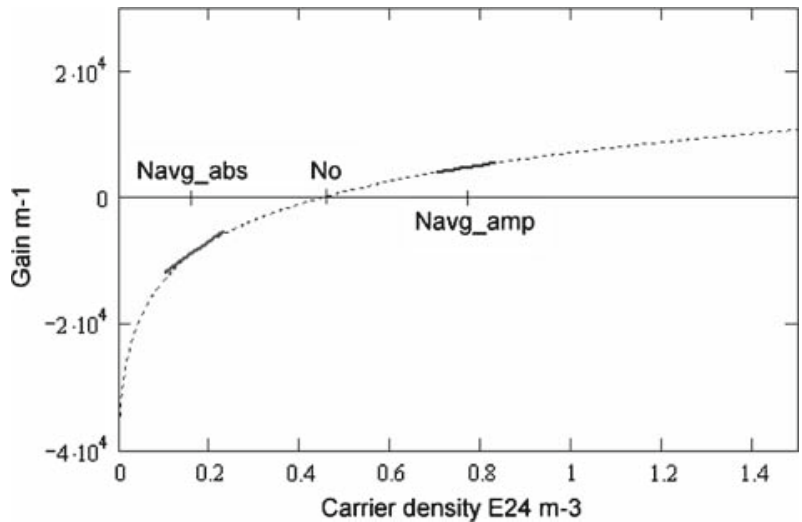

Fig. 2 Plot of the logarithmic gain carrier density relation. The average carrier densities in the amplifier and the SA in a modelocked ring laser are indicated

$$
g_{m}=a N \cdot N_{0} \cdot \ln \left(\frac{N}{N_{0}}\right)
$$

Each SOA segment is described by the following rate equations:

$$
\begin{aligned}
\frac{\partial S_{1}}{\partial t}= & S_{1} \cdot \frac{C_{S_{0}} \cdot \ln \left(\frac{N}{N_{0}}\right)-C_{S_{1}} \cdot\left(S_{1}+S_{2}\right)^{2}}{1+C_{2} \cdot\left(S_{1}+S_{2}\right)}-C_{S 3} \cdot S_{1}^{2} \\
& -\operatorname{Loss}_{1} \cdot S_{1}-\operatorname{Loss}_{2} \cdot N \cdot S_{1}+C_{S_{4}} \cdot N^{2}
\end{aligned}
$$




$$
\begin{aligned}
& \frac{\partial S_{2}}{\partial t}=S_{2} \cdot \frac{C_{S_{0}} \cdot \ln \left(\frac{N}{N_{0}}\right)-C_{S_{1}} \cdot\left(S_{1}+S_{2}\right)^{2}}{1+C_{2} \cdot\left(S_{1}+S_{2}\right)}-C_{S_{3}} \cdot S_{2}^{2} \\
& -\operatorname{Loss}_{1} \cdot S_{2}-\operatorname{Loss}_{2} \cdot N \cdot S_{2}+C_{S_{4}} \cdot N^{2} \\
& \frac{\partial N}{\partial t}=-\left(S_{1}+S_{2}\right) \cdot \frac{C_{N_{0}} \cdot \ln \left(\frac{N}{N_{0}}\right)-C_{N_{1}} \cdot\left(S_{1}+S_{2}\right)^{2}}{1+C_{2} \cdot\left(S_{1}+S_{2}\right)} \\
& +C_{N_{3}} \cdot\left(S_{1}+S_{2}\right)^{2}-A \cdot N-B \cdot N^{2}-C \cdot N^{3}+W p \\
& \frac{\partial \varphi_{1}}{\partial z}=\frac{C_{\varphi_{0}} \cdot \ln \left(\frac{N}{N_{0}}\right) \cdot\left(S_{1}+S_{2}\right)+C_{\varphi_{1}} \cdot\left(S_{1}+S_{2}\right)^{2}}{1+C_{2} \cdot\left(S_{1}+S_{2}\right)} \\
& -C_{\varphi_{3}} \cdot \ln \left(\frac{N}{N_{0}}\right)-C_{\varphi_{4}} \cdot\left(S_{1}+S_{2}\right) \\
& \frac{\partial \varphi_{2}}{\partial z}=\frac{C_{\varphi_{0}} \cdot \ln \left(\frac{N}{N_{0}}\right) \cdot\left(S_{1}+S_{2}\right)+C_{\varphi_{1}} \cdot\left(S_{1}+S_{2}\right)^{2}}{1+C_{2} \cdot\left(S_{1}+S_{2}\right)} \\
& -C_{\varphi_{3}} \cdot \ln \left(\frac{N}{N_{0}}\right)-C_{\varphi_{4}} \cdot\left(S_{1}+S_{2}\right)
\end{aligned}
$$

Here $S_{1}$ and $S_{2}$ are the CW and CCW photon densities, $\phi_{1}$ and $\phi_{2}$ are the CW and CCW phases. In the two photon density equations for $\mathrm{CW}$ (2) and $\mathrm{CCW}$ propagation (3): $C_{S_{0}}$ gives the linear gain; $C_{S_{1}}$ gives the nonlinear gain compression due to two-photon absorption; $C_{2}$ gives the nonlinear gain compression due to spectral hole burning and carrier heating; $C_{S_{3}}$ gives the two-photon absorption; Loss $s_{1}$ is the scattering loss and the free carrier loss in the cladding; $\operatorname{Loss}_{2}$ is the free carrier absorption within the active layer (Williamson and Adams 2004); $C_{S_{4}}$ is the bimolecular recombination contribution. In the carrier density equation: $C_{N-0}, C_{N_{1}}, C_{N_{3}}$ and $C_{2}$ are similar contributions as $C_{S_{0}}, C_{S_{1}}, C_{S_{3}}$ and $C_{2} ; A$ is the inverse of the carrier lifetime; $B$ is the bimolecular recombination rate; $C$ is the Auger recombination coefficient and $W p$ is the pump rate. In the two phase equations: $C_{\phi_{0}}, C_{\phi_{1}}, C_{\phi_{3}}$ and $C_{2}$ are the same contributions as $C_{S_{0}}, C_{S_{1}}, C_{S_{3}}$ and $C_{2}, C_{\phi_{4}}$ is the UNR coefficient.

In detail:

$$
\begin{aligned}
& C_{S_{0}}=a N \cdot \Gamma \cdot V g \cdot N_{0} \\
& C_{\varphi_{0}}=\frac{1}{2} \cdot \alpha T \cdot \varepsilon_{1} \cdot a N \cdot \hbar \cdot \omega \cdot \operatorname{Vg} \cdot \operatorname{Surf} \cdot N_{0} \\
& C_{S_{1}}=\varepsilon_{2} \cdot \hbar^{2} \cdot \omega^{2} \cdot V g^{3} \cdot \frac{\operatorname{Surf}^{2}}{\Gamma^{2}} \\
& C_{\varphi_{1}}=\frac{1}{2} \cdot \alpha T \cdot \varepsilon_{2} \cdot \hbar^{2} \cdot \omega^{2} \cdot V g^{2} \\
& C_{2}=\varepsilon_{1} \cdot \hbar \cdot \omega \cdot V g \cdot \frac{\operatorname{Surf}}{\Gamma} \\
& C_{S_{3}}=\Gamma_{2} \cdot \beta_{2} \cdot \hbar \cdot \omega \cdot V g^{2} \\
& C_{\varphi_{3}}=\frac{1}{2} \cdot \alpha N \cdot a N \cdot \Gamma \cdot V g \cdot N_{0} \\
& C_{N_{0}}=a N \cdot V g \cdot N_{0} \\
& \text { Loss }_{1}=\frac{\left(\text { Loss }_{s} \text { cat }+ \text { Loss }_{\text {clad }}\right) \cdot L_{\text {seg }}}{T_{\text {seg }}} \\
& C_{S_{4}}=B \cdot \Gamma \cdot \beta \\
& C_{\varphi_{4}}=\Gamma_{2}^{\prime} \cdot \frac{\omega}{c} \cdot n_{2} \cdot \hbar \cdot \omega \cdot V g \\
& \mathrm{C}_{N_{1}}=\varepsilon_{2} \cdot \hbar^{2} \cdot \omega^{2} \cdot \operatorname{Vg}^{3} \cdot \frac{\operatorname{Surf} f^{2}}{\Gamma^{3}} \\
& C_{N_{3}}=\frac{\Gamma_{2}}{\Gamma} \cdot \beta_{2} \cdot \hbar \cdot \omega \cdot V g^{2} \\
& \operatorname{Loss}_{2}=\frac{\text { Loss }_{f r e e c a r} \cdot L_{\text {seg }}}{T_{\text {seg }}}
\end{aligned}
$$

Where $\Gamma$ the linear confinement factor, $V g$ the group velocity, $\varepsilon_{2}$ the nonlinear gain compression factor to TPA, $\omega$ the photon frequency, $\varepsilon_{1}$ the nonlinear gain compression factor, Surf 
Table 1 Parameters used in the model

\begin{tabular}{lll}
\hline Symbol & Description & Value \\
\hline$a N$ & Linear gain coefficient & $4.05 \cdot 10^{-20} \mathrm{~m}^{2}$ \\
$N_{0}$ & Transparency carrier density & $0.4 \cdot 10^{24} \mathrm{~m}^{-3}$ \\
$\Gamma$ & Linear confinement factor & 0.259 \\
$\Gamma_{2}$ & Confinement factor for TPA & 0.5 \\
$\Gamma_{2}^{\prime}$ & Confinement factor for UNR & 0.4 \\
$V g$ & Group velocity & $80 \cdot 10^{6} \mathrm{~m} / \mathrm{s}$ \\
$\varepsilon_{1}$ & Nonlinear gain compression factor & $0.2 \mathrm{~W}^{-1}$ \\
$\varepsilon_{2}$ & Nonlinear gain compression factor to TPA & $200 \mathrm{~W}^{-2} \mathrm{~m}^{-1}$ \\
$\omega$ & Pulsation / wavelength & $1.55 \mu \mathrm{m}$ \\
Surf & Surface of the active region & $0.12 \times 2.0 \mu \mathrm{m}^{2}$ \\
$n_{2}$ & Nonlinear gain refractive index & $-3.5 \cdot 10^{-16} \mathrm{~m}^{2} / \mathrm{W}$ \\
$T_{S e g}$ & Time segment & $25 \mathrm{fs}$ \\
$\beta$ & Spontaneous emission coupling factor & $10^{-5}$ \\
$\beta_{2}$ & Coefficient for TPA & $37 \mathrm{~cm}^{-5} \mathrm{GW}^{-1}$ \\
$A$ & One over the carrier life time & $1.67 \cdot 10^{-9} \mathrm{~s}^{-1}$ \\
$B$ & Bimolecular recombination rate & $2.602 \cdot 10^{-16} \mathrm{~m}^{3} \mathrm{~s}$ \\
$C$ & Auger recombination coefficient & $5.269 \cdot 10^{-41} \mathrm{~m}^{6} \mathrm{~s}$
\end{tabular}

the surface of the active region, $\Gamma_{2}$ the confinement factor for TPA, $\Gamma_{2}^{\prime}$ the confinement factor for UNR, c the speed of light, $T_{\text {seg }}$ the time segment, $L_{\text {seg }}$ the physical length of one segment, $\beta$ the spontaneous emission coupling factor, $\alpha N$ the carrier density linewidth enhancement factor and $\alpha T$ is the temperature linewidth enhancement factor. $\beta_{2}$ is the coefficient for twophoton absorption and $n_{2}$ is the nonlinear gain refractive index. All the parameters used are listed in Table 1.

The SA is a short SOA that is reversely biased (Lau and Paslaski 1991). It is described with the same rate equations as the amplifier without carrier injection. In the SA in an operating MLL the carrier density varies in time between low background values and the transparency carrier density value. In Fig. 2, it can be seen that within this range, the slope of the gaincarrier relation curve varies by a significant amount. The logarithmic gain-carrier relation has its limit when the carrier density in the SA is very low. The absorption then becomes unrealistically high. This is prevented by introducing a minimum carrier density background value in the SA. It has been reported that the carrier lifetime of a SA depends of the reverse bias voltage applied (Karin et al. 1994), but such a relation has not been implemented in the model. The carrier lifetime is the only parameter that is tuned. The model presented here is bidirectional, thus two pulses can meet in the SA. For a very short SA (less than a few wavelengths), coherent effects need to be taken into account. However for MLLs with ridge waveguides, the coherent effects are small in the SA (Derickson et al. 1992) and therefore have not been implemented in the model. The isolation sections between an amplifier and a SA are considered as non-reversely biased SAs with a fixed carrier lifetime of $200 \mathrm{ps}$ and no current injection. In passive waveguides, only the passives losses are taken into account. Intensities of pulses are not sufficiently high to introduce non linear effects in the passive waveguides (Van Thourhout et al. 2001).

In this paper, a RMLL cavity where the two counter propagating pulses experience the same optical path, but in opposite direction, is called a symmetric cavity. If not, the cavity is called 
asymmetric. Besides, a modelocked laser which has two counter propagating pulses meeting in a SA, is in a colliding pulse modelocked (CPM) (Bischoff et al. 1997) configuration. This is automatically the case for a RMLL (in case there are no provisions to make it unidirectional). Shorter pulses are generally obtained with such a configuration (Bischoff et al. 1997). Figure 1 presents a diagram of a possible RMLL symmetric configuration. At the top of the figure, a coupler element couples the light in and out of the ring. This is done coherently for both directions in the model. At the bottom of the figure, in the middle of an amplifier is a SA isolated by two isolation sections. The white segments are passive waveguides. The bandwidth limitation obtained by filtering is done for each direction just before the light is coupled out of the ring.

\subsection{Bandwidth limitation using a digital Bessel filter}

The effect of the limited optical bandwidth of the SOA has not been included in the rate equations of the SOA presented above. The limitation of the optical bandwidth is brought separately at each roundtrip using a bi-directional digital low-pass filter (Hamming 1989). This method has the advantage that the model is fully in the time domain. The filter parameters have been set so that the transmission curve is close to the measured gain curve (Barbarin et al. 2006) as can be seen from Fig. 3a. The bandwidth of the SOA has been measured to be $21.6 \mathrm{THz}$ ( 0.086 in a normalized frequency). Important is also that such a digital Bessel filter introduces a very low distortion of the phase. The filter is a digital difference Eq. 7 . The difference equation computes the output electrical field strength and phase at the time $\mathrm{n}$, based on past and present input electrical field and past output electrical field.

$$
y[n]=\sum_{k=0}^{\text {order }} b_{k} \cdot x[n-k]+\sum_{l=1}^{\text {order }}-a_{l} \cdot y[n-k]
$$

In Eq. 7, $x$ [ ] is the array with the present and past input signal (complex field amplitude), $y$ [ ] is the array with the past output signal (complex field amplitude), $\boldsymbol{a}$ is a vector of filter feedback coefficients, and $\boldsymbol{b}$ is a vector of filter feedforward coefficients. These coefficients determine the properties of the filter. One possible signal flow graph for the filter is given in Figure $3 \mathrm{~b}$. The storage of past samples is represented by boxes labeled " $\mathrm{Z}$ "-1". This diagram is used because it can be directly converted to the frequency domain by replacing all timedomain signals by their respective $Z$ transforms (Jury 1973). The idea contained within the Z-transform was previously known as the "generating function method".

The MathCad ${ }^{\circledR}$ software package has been used to calculate the feedback and feedforward coefficients of the Bessel filter. The 14th order for the filter turned out to be sufficiently robust for our application. In the calculations the $\boldsymbol{a}$ and $\boldsymbol{b}$ coefficients need to be used with an accuracy of that is two orders of magnitude greater than the order of the filter. This type of digital filter conserves the causality of the signal but it has a delay. The delay is one over the normalized frequency cut, which in our case corresponds to approximately 22 segments of $25 \mathrm{fs}$. This delay can be compensated in the passive region of the ring where only the passive losses are playing a role. The use of a digital filter is much more simple than using a Fourier Transform (FT), spectral filtering and a back FT.

\section{Simulation of symmetric and asymmetric $20 \mathrm{GHz}$ ring modelocked lasers}

The model has been used to study a symmetric and an asymmetric $20 \mathrm{GHz}$ RMLL design. The results are presented in the two following sections. These RMLL designs can be realized with 
a)

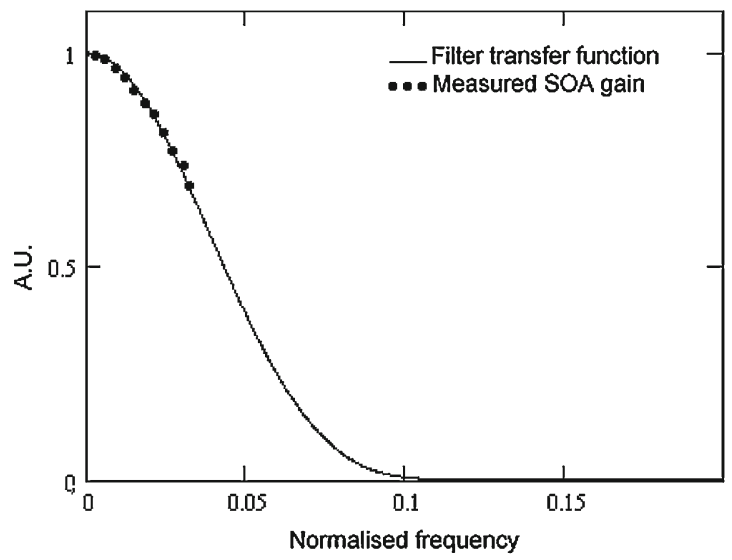

b)

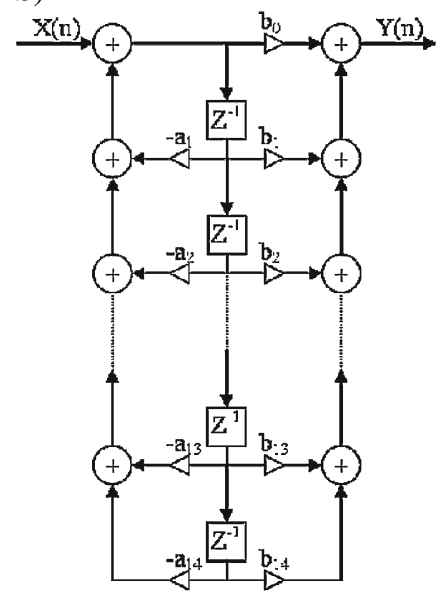

Fig. 3 (a) The transfer function of the digital filter used, plotted with the measured bulk SOA gain as a function of the normalized frequency $\left(\Omega=\omega \cdot T_{\text {seg }} / 2 \pi\right)$. (b) Signal flow graph of a digital filter. " $Z^{-1}$ " represents a Z-Transform. "a" and "b" are the feedback and feedforward coefficients of the filter

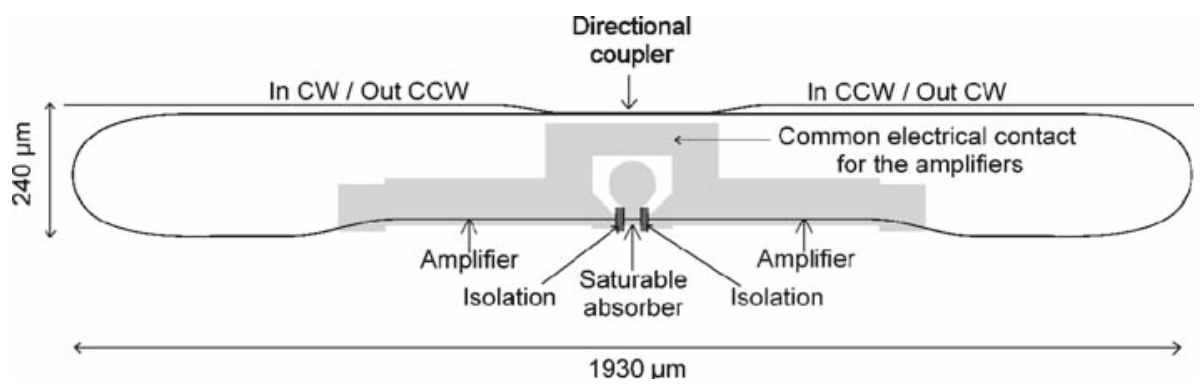

Fig. 4 Design of a symmetric $20 \mathrm{GHz}$ modelocked ring laser on an active passive wafer. The active region is $1000 \mu \mathrm{m}$ long and centred is a $22 \mu \mathrm{m}$ long SA. The isolation sections are $16 \mu \mathrm{m}$ long

the COBRA technology (Bente and Smit 2006). The choice of the design parameters studied has been guided by the results obtained experimentally from an $18 \mathrm{GHz}$ integrated extended cavity Fabry-Pérot MLL (EC-FPMLL) (Barbarin et al. 2007). Indeed the dimensions of the RMLL amplifier and SA studied are similar to the experimental design of the EC-FPMLL.

\subsection{Simulation of a symmetric RMLL}

The design of a $20 \mathrm{GHz}$ symmetric RMLL is plotted in Fig. 4. The total length of the ring laser is $4 \mathrm{~mm}$ (2000 segments). The two amplifiers are $472 \mu \mathrm{m}$ long (236 segments). The choice of the length of the SA is based on the length of the SAs in successfully modelocked FPMLL devices (Barbarin et al. 2005b): absorber lengths of 10 and $12.5 \mu \mathrm{m}$. In a ring cavity, there are two counter-propagating pulses which meet in the SA. It can be seen as two FPMLL lasers joined at the SAs; the resulting SA is then two times as long. For the simulation, the SA was set at $22 \mu \mathrm{m}$ long (11 segments). It is isolated from the amplifiers by two $16 \mu \mathrm{m}$ long (8 segments) isolation sections. The light is coupled in and out from the ring with a coupler with $50 \%$ out-coupling ratio. 


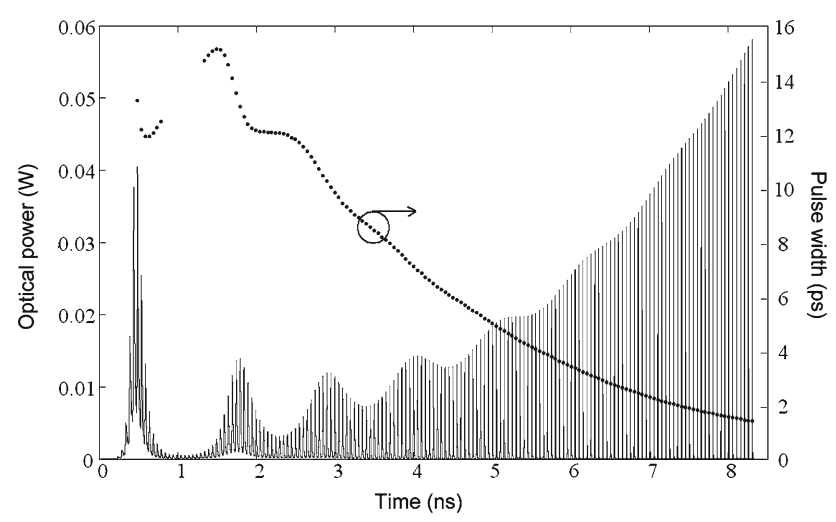

Fig. 5 Simulated output power and calculated pulse width of the laser as a function of time. The laser is self-starting. I $=65 \mathrm{~mA} \tau_{\mathrm{SA}}=11 \mathrm{ps}$

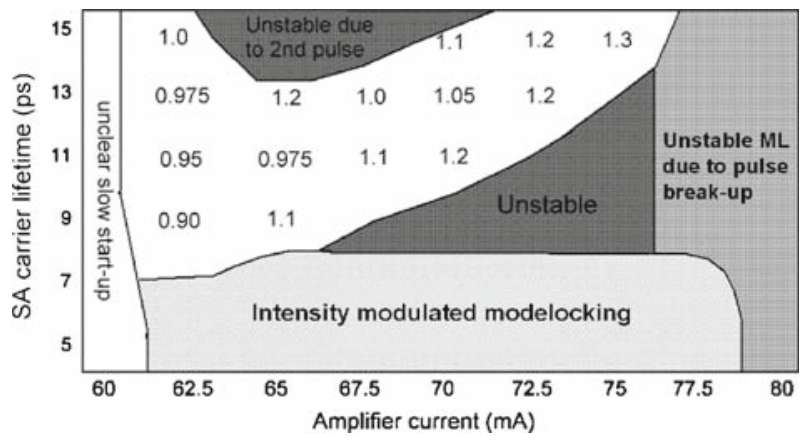

Fig. 6 Different regimes of the symmetric $20 \mathrm{GHz}$ RMLL function of amplifier current and SA carrier lifetime. The pulse width is given in picoseconds if the laser is modelocked

As the model includes spontaneous emission, the self-starting of the laser can be simulated. As an example, a simulated output power during start-up of the laser is plotted in Fig. 5 for settings that lead to a stable modelocked state. Three aspects can be identified in the start-up of the modelocked laser. The laser starts with relaxation oscillation behaviour which ends after five or so oscillations. The second aspect is that the modelocking action of the absorber can be seen right from the start and after only $2-3$ ns the laser produces pulses and only a small amount of CW background light. Then the pulse length decreases further in time as shown in Fig. 5 (right axis). After that the third aspect can be seen. The short pulse length makes that the self-phase modulation becomes important. The short pulse may break-up a couple of times before its phase profile becomes stable. The laser then settles to a stable modelocked state.

Simulations of the symmetric RMLL have been performed for different amplifier current and SA carrier lifetime values to establish the stability region for modelocking. To save computing time, two low power 2 ps pulses with a null phase have been injected into the cavity to start the simulations. For a limited number of working points this has been tested to lead to the same results as starting from only the ASE. The laser threshold is $57.5 \mathrm{~mA}$. The different regimes of the laser are plotted in Fig. 6. The laser is properly modelocked for amplifier current values between 62 and $75 \mathrm{~mA}$ and SA carrier lifetime values between 9 and $15 \mathrm{ps}$. The results obtained for parameters in the modelocked regime on the boundary of 

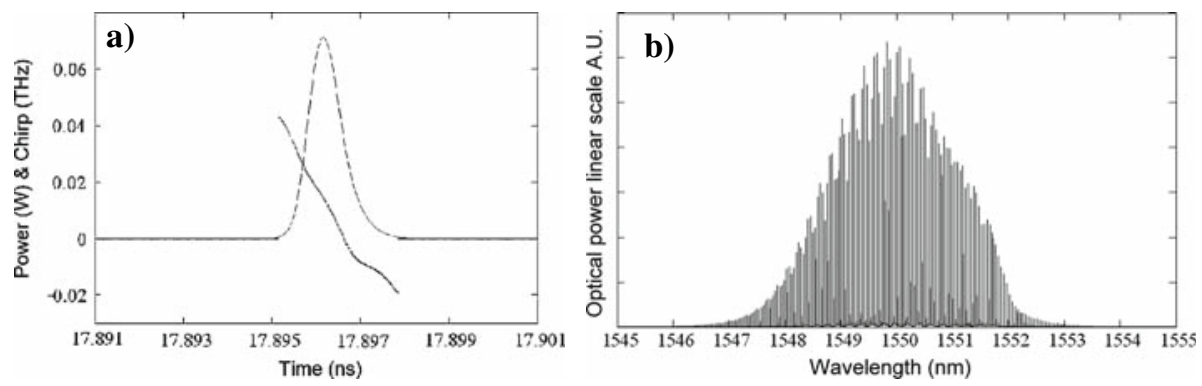

Fig. 7 (a) Simulated output pulse shape and chirp and (b) the corresponding calculated optical spectrum. Width $=0.95 \mathrm{ps}$, Peak power $=70 \mathrm{~mW}$; Chirp $=65 \mathrm{GHz}(\sim$ linear $)$; Bandwidth $=2.6 \mathrm{~nm}$; for $\mathrm{I}=65 \mathrm{~mA} \&$ $\tau_{\mathrm{SA}}=11 \mathrm{ps}$

the stability region are stable over $5 \mathrm{~ns}$. At high current injection $(>75 \mathrm{~mA})$ and for any SA carrier lifetime, the modelocking in the laser becomes unstable due to pulse break-up, but in a regular way. For short SA carrier lifetime $(<7 \mathrm{ps})$ and a current in the amplifier which is not too high, the laser goes into an intensity modulated modelocked state. This regime is a two dimensional quasi-periodic dynamic (Ott 1993). There are two other regions where the laser is also unstable. In the first unstable region around $65 \mathrm{~mA}$ amplifier current and 15 ps SA carrier lifetime, a second pulse in the roundtrip time attempts to be present. In the second region unstable around $72.5 \mathrm{~mA}$ amplifier current and $9 \mathrm{ps}$ SA carrier lifetime, the laser switches irregularly between a pulse break-up regime and more stable modelocked state. Each regime is studied in more detail in the following paragraphs.

In the stable modelocking region, as can be seen in Fig. 6, the pulse width increases with the current, except next to the unstable region. At lower current, the pulse is less intense; it saturates less the SA which vice-versa narrows down the pulse more efficiently. In the same way the pulse width decreases with the carrier lifetime of the SA, except close to the unstable regimes. The most stable modelocked regime (over $20 \mathrm{~ns}$ simulated) has been found for $65 \mathrm{~mA}$ amplifier current and $11 \mathrm{ps}$ SA carrier lifetime. The measured pulse width is constant after $8 \mathrm{~ns}$ and the amplitude modulation is small $(<10 \%)$. The output pulse obtained is plotted in Fig. 7a together with the corresponding calculated optical spectrum in Fig. 7b. The pulse is 0.95 ps wide with a $70 \mathrm{~mW}$ peak power and it is slightly down-chirped $(65 \mathrm{GHz})$. Unidirectional simulations of Heck et al. (2006) predicted up-chirped pulses with higher chirp values. The low values and sign change found in the model here, is attributed to the CPM configuration. Using a Fourier Transform (FT), the optical spectrum has been calculated with 100 pulses. The calculated spectrum is $2.6 \mathrm{~nm}$ at FWHM: the pulse is near transform-limited. The chirp of the pulses stays limited due to the extended cavity.

The two-dimensional quasi-periodic regime at a short SA recovery time $(<7 \mathrm{ps})$ is illustrated by the simulated output power of the laser in Fig. 8 . This regime is stable after $5 \mathrm{~ns}$. The peak intensities of the pulses vary from 120 to $60 \mathrm{~mW}$ every $1.3 \mathrm{~ns}(770 \mathrm{MHz})$. In the same way, the pulse width varies from 0.75 to $1.05 \mathrm{ps}$. The narrowest and the widest pulses are plotted in Fig. 9a and b. In both cases the chirp is small $(<60 \mathrm{GHz})$ and there is a down-chirp. The evolution of the pulse width in time is shown in Fig. 10. In this regime the SA recovers faster and the absorption is higher. The amplitude modulation can be reduced by increasing the gain in the cavity. Indeed, simulations with a higher injection current in the amplifier show that the modulation decreases and pulses narrow down. But, if the current is increased further, pulses start to break-up. No proper modelocking without amplitude modulation has been simulated for this design at such a short SA recovery time. 


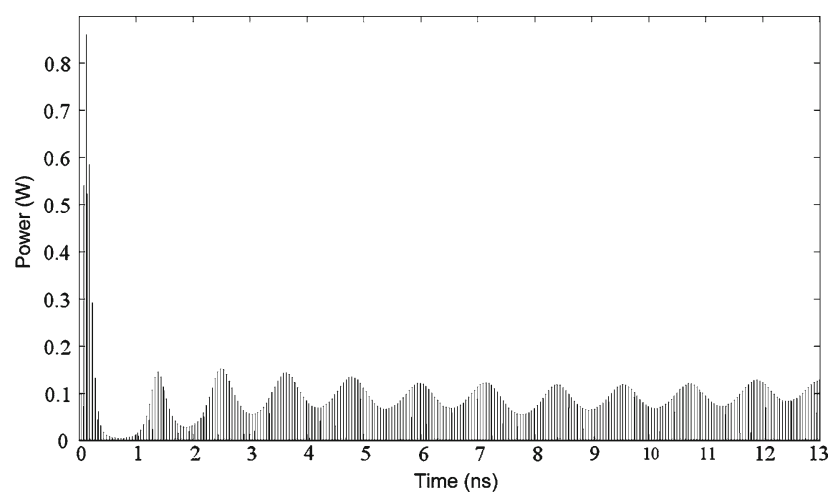

Fig. 8 Simulated output power of the $20 \mathrm{GHz}$ symmetric RMLL as a function of time with a relatively short SA lifetime. Two low power 2 ps pulses with a phase null were injected in both direction of the cavity to speed up the start of the laser. $\mathrm{I}=67.5 \mathrm{~mA} \tau_{\mathrm{SA}}=5 \mathrm{ps}$
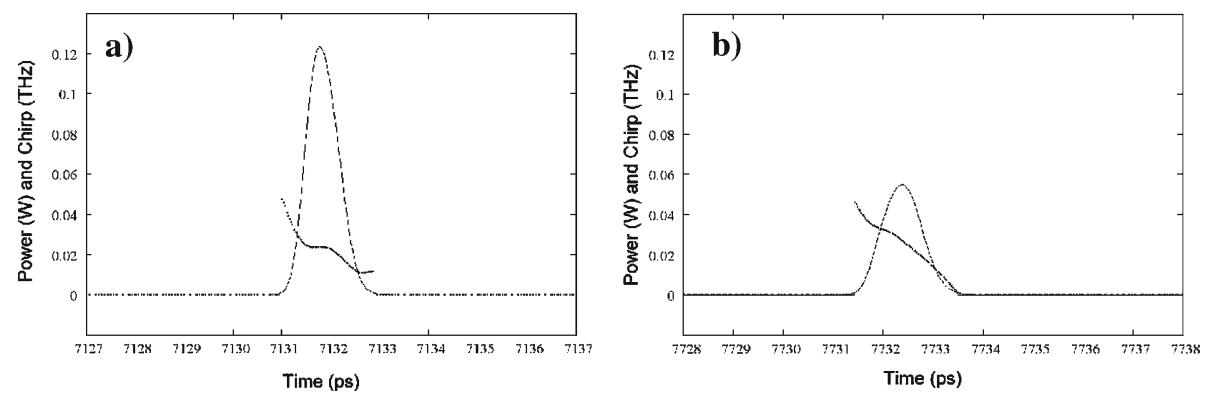

Fig. 9 Simulated output pulses for the two extreme cases, $\mathrm{I}=67.5 \mathrm{~mA} \tau_{S A}=5 \mathrm{ps}$ : (a) Peak power $=$ $122 \mathrm{~mW}$, width $=0.75 \mathrm{ps}$, down chirp $=30 \mathrm{GHz}$ (nonlinear) $(\mathbf{b})$ Peak power $=58 \mathrm{~mW}$, width $=1.05 \mathrm{ps}$, down chirp $=50 \mathrm{GHz}(\sim$ linear $)$

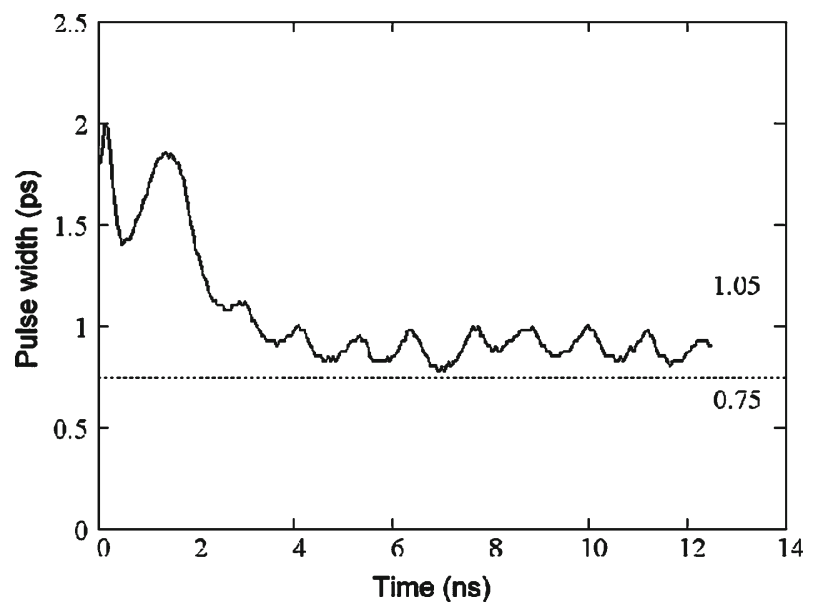

Fig. 10 Simulated pulse width function of time for $\mathrm{I}=67.5 \mathrm{~mA} \tau_{\mathrm{SA}}=5 \mathrm{ps}$ 


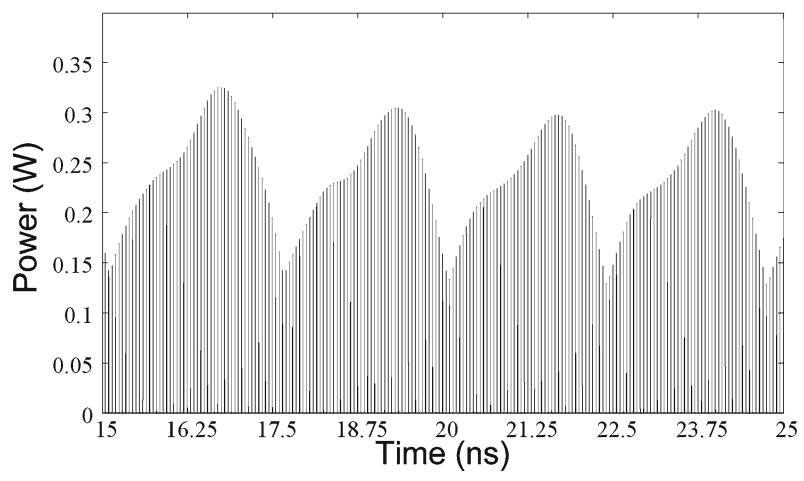

Fig. 11 Simulated output power of the $20 \mathrm{GHz}$ symmetric RMLL as a function of time at higher current values. Two low power 2 ps pulses with a phase null were injected in both direction of the cavity to speed up the start of the laser. $\mathrm{I}=80 \mathrm{~mA} \tau_{\mathrm{SA}}=7 \mathrm{ps}$

Longer timescale variations in output power are observed in combination with short pulse output with repetitive pulse break-up. These phenomena that occur in the modelocked laser at high injection current (over $76 \mathrm{~mA}$ in this design) can be understood by analyzing the simulated time series in detail. The short pulses tend to become more intense and narrower. This makes that the short pulses start to break up. In some cases there is a continuous cycle of the short pulse breaking up, then a new similar short pulse emerges which in turn breaks up again, and this continues. An example of such behaviour is shown in Fig. 11 over a $10 \mathrm{~ns}$ span. The pattern repeats itself every $2.55 \mathrm{~ns}(450 \mathrm{MHz})$ in this example. The envelope of the time series is not a single frequency oscillation (Ott 1993), thus this regime is a quasi periodic dynamic state with high dimension ( $>3$ ). The evolution of the pulse break up and subsequent emerging of another pulse from the tail of the initial pulse during the $2.55 \mathrm{~ns}$ period is shown in Fig. 12.

The unstable region at the top of Fig. 6 where the SA carrier lifetime is $15 \mathrm{ps}$ and the current lies between 64 and $68 \mathrm{~mA}$, can be explained. At such SA carrier lifetime, the absorption is not always sufficiently high to prevent a second pulses to build up. A second gain window appears after the first pulse due to the relatively slow absorber recovery with respect to the roundtrip-time. This makes the regime unstable (see Fig. 13). For the same carrier lifetime, at around $62 \mathrm{~mA}$ in the amplifier, the gain is not sufficient to supply a second pulse, thus it is stable. At $65 \mathrm{~mA}$ there is sufficient gain to have a small second pulse in the tail of the first pulse which makes the laser unstable. Above $70 \mathrm{~mA}$, the two counter propagating pulses in the cavity are more intense and deplete the gain in the amplifier more deeply. Therefore no other pulses can build up.

The influence of the non-linear effects in the equations on the zone of stable modelocking was studied for the symmetric laser design. In the optimal modelocking point, removing the two photon effects (absorption and gain compression effects) and non-linear refractive index, led to very much the same output only at a slightly higher output power. When going to higher current values effects of pulse break-up (Fig. 6) also do occur but at somewhat higher currents (in the order of $5 \mathrm{~mA}$ typically). Similarly the modulated modelocking at low SA lifetimes also still occurs but now at lifetimes of 6 to $6.5 \mathrm{ps}$ instead of 7 to $7.5 \mathrm{ps}$. Thus removing these non-linear effects leads to an enlarged stability region for modelocking in the simulation. The differences are relevant but not particularly large. 


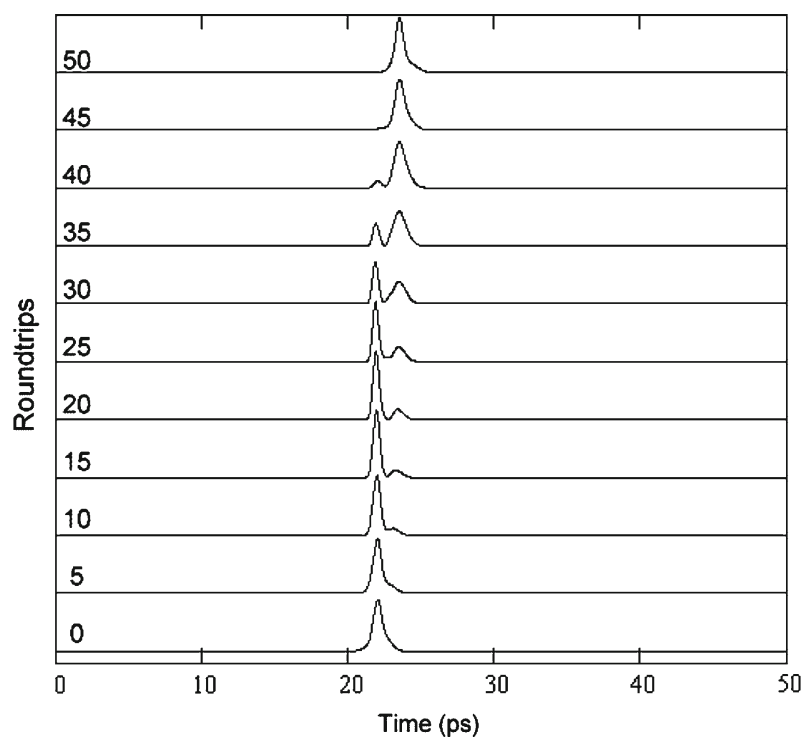

Fig. 12 Pulse break-up evolution. The pulse shape in shown for a series of roundtrips. $\mathrm{I}=80 \mathrm{~mA} \tau_{\mathrm{SA}}=7 \mathrm{ps}$

Fig. 13 Simulated output power of the $20 \mathrm{GHz}$ symmetric RMLL in $\mathrm{dB}$ scale as function of time for one selected roundtrip, $\tau_{\mathrm{SA}}=15 \mathrm{ps}$. Depicted are the results for three different amplifier currents

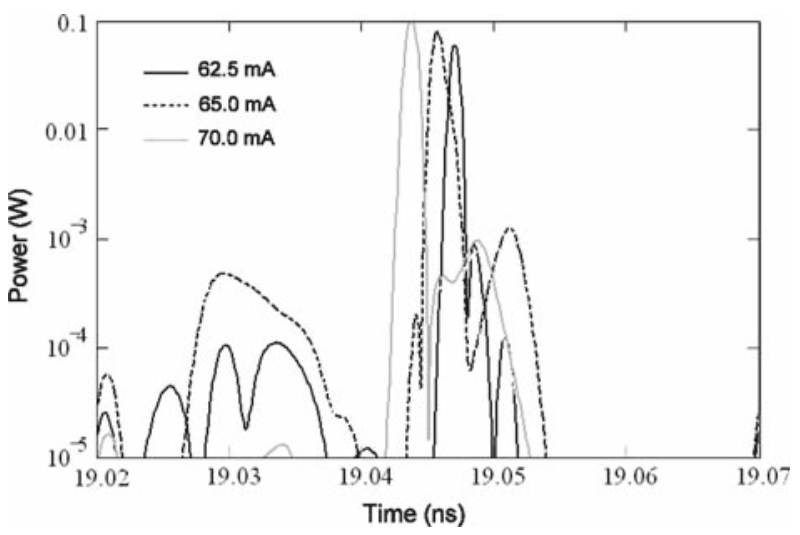

\subsection{Simulation of an asymmetric RMLL}

The next laser design studied is asymmetric for the two counter-propagating pulses, and is depicted in Fig. 14. When two counter-propagating pulses are in the cavity, they meet in the SA and in the passive waveguide at the right-hand side of the coupler. The CCW pulse enters the SOA which has just been depleted by the CW pulse. Such a design is intended to investigate the possibility of having an integrated RMLL operating in a unidirectional regime since there is a roundtrip gain difference expected for the pulses in the two directions. It is of interest to achieve unidirectional operation without the use of an optical isolator which is not 


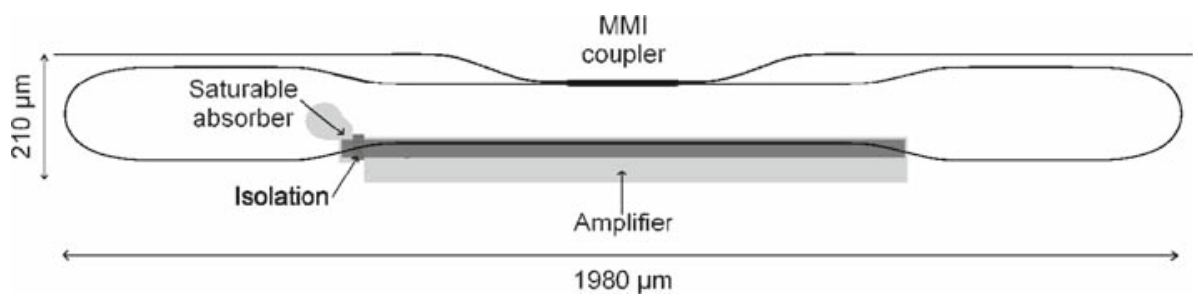

Fig. 14 Design of an asymmetric $20 \mathrm{GHz}$ modelocked ring laser on an active passive wafer. The active region is $1000 \mu \mathrm{m}$ long, at the side is a $22 \mu \mathrm{m}$ long SA. The single isolation section is $16 \mu \mathrm{m}$ long

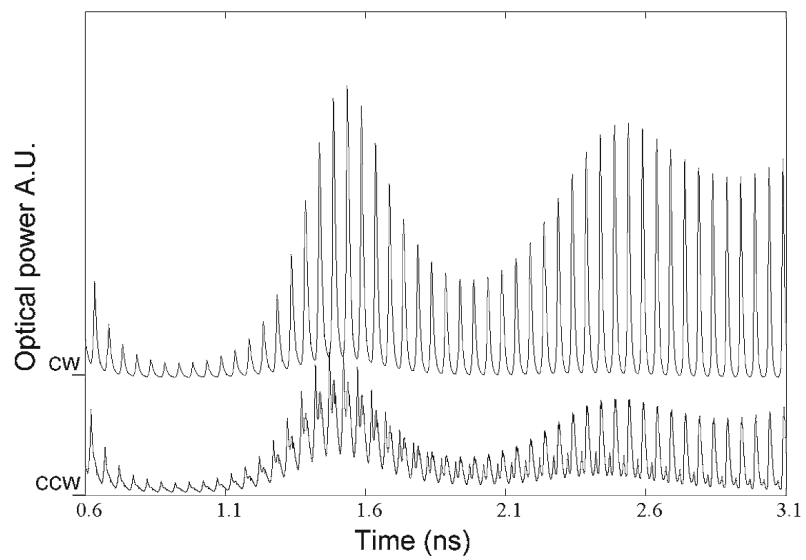

Fig. 15 Simulated output power $(\mathrm{CW}$ and $\mathrm{CCW})$ of the laser as a function of time. The time reference is the $\mathrm{SA}$. In the CCW direction a pulse builds-up that is synchronized to the $\mathrm{CW}$ pulse, both pulses meet in the SA. $\left(\mathrm{I}=65 \mathrm{~mA} \tau_{\mathrm{SA}}=11 \mathrm{ps}\right)$

available for integration at the moment or without external feedback. The SA is positioned at the side of the amplifier. A $16 \mu \mathrm{m}$ long ( 8 segments) section isolates the SA from the amplifier. The length of the SA is the same as the symmetric device studied in the previous section: $22 \mu \mathrm{m}$ (11 segments). The simulation results show that depending on the driving conditions of the RMLL, the laser can have two counter-propagating pulses with different intensities or operates in a near-unidirectional regime.

The simulation of the self-starting of the laser gave an interesting result. The output power levels of the laser in the two directions as a function of time are plotted in Fig. 15. Here the time axis has been shifted for one of the output signals such that when two pulses in the two plotted output channels coincide, the two pulses travelling inside the laser cavity overlap in the SA. In this example, the RMLL supports two counter-propagating pulses in the cavity. The long time scale modulation of the $\mathrm{CW}$ and $\mathrm{CCW}$ output powers is similar to the one in the symmetric case that has been presented in Fig. 5. However in the asymmetric case the building up of the pulses is different. First, it can be seen that the $\mathrm{CW}$ output power is more powerful than the $\mathrm{CCW}$ one. Then, the $\mathrm{CW}$ pulse builds-up in the same way as the pulses in the symmetric design, but not the CCW pulse. The initial "coarse" CCW pulse is not synchronized in the SA with the CW pulse. As the CW pulse is more powerful, it suppresses the $\mathrm{CCW}$ pulse, but a second $\mathrm{CCW}$ pulse builds-up that overlaps with the $\mathrm{CW}$ pulse in the SA. At the end, the laser has two counter-propagating pulses with different intensities. 


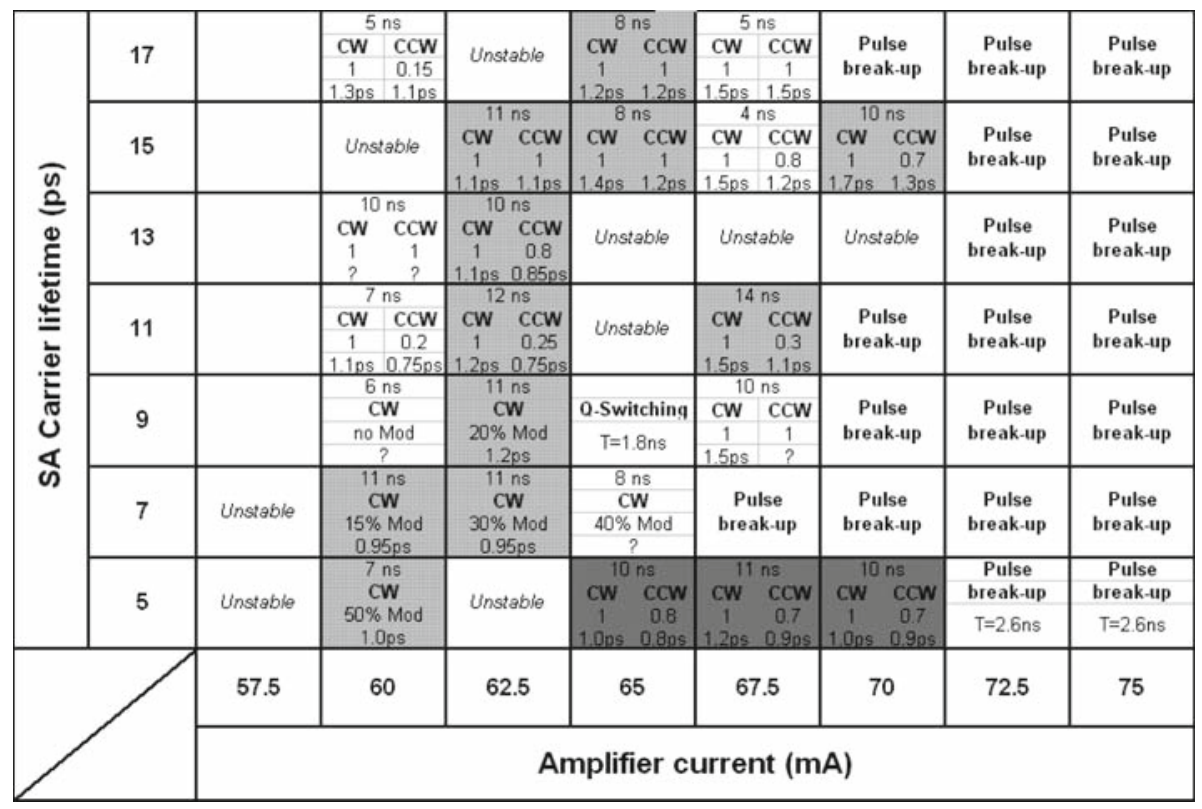

Fig. 16 Different regimes of the asymmetric $20 \mathrm{GHz}$ RMLL as function of amplifier current and SA carrier lifetime. If the laser is modelocked the ratio between the $\mathrm{CW}$ and $\mathrm{CCW}$ pulses is mentioned with the pulses width

Simulations of the asymmetric RMLL have been performed for different amplifier current values and SA carrier lifetimes similarly as in Sect.3.1 For this design, the lasing threshold is $55 \mathrm{~mA}$. The first general observation is that, this configuration is much less stable than the symmetric design. The instability originates directly from the competition between the $\mathrm{CW}$ and the $\mathrm{CCW}$ modes. Amplifier current and SA carrier lifetime settings that gave rise to states which showed stable behaviour over $5 \mathrm{~ns}$ have been analyzed further. These operating points are presented in Fig. 16. For operating points where two counter-propagating pulses are supported, the ratio in peak power and the pulse widths for the two directions are indicated.

Different modes of operation can clearly be seen. Like the symmetric device simulation results, the pulse widths of the counter-propagating pulses increase with the current in the amplifier and decrease with $\tau_{\mathrm{SA}}$. The chirp of the pulses stays limited due to the integrated extended cavity. At high current injection $(>70 \mathrm{~mA})$ and for all simulated carrier lifetime values the laser shows pulse break up and related variations in peak power intensity, just as the symmetric device. For a relatively short SA carrier lifetime $(<9 \mathrm{ps})$ and a current in the amplifier which is not too high $(<65 \mathrm{~mA})$, the laser produces short pulses modulated in intensity. The modulation depth of the envelope is indicated as a percentage in Fig. 16. This modulation increases at higher amplifier current and at lower SA carrier lifetime.

The most stable states are indicated as the light grey areas in Fig. 16. From the location of these areas the conclusion can be drawn that the SA carrier lifetime $\left(\tau_{\mathrm{SA}}\right)$ is the most important parameter to control the asymmetry between the $\mathrm{CW}$ and $\mathrm{CCW}$ output pulses. At low $\tau_{\mathrm{SA}}(<9 \mathrm{ps})$ the laser operates in a near-unidirectional regime as shown in Fig. 17a. The resulting pulses are plotted in Fig. 18 for $\mathrm{I}=60 \mathrm{~mA} \tau_{\mathrm{SA}}=8 \mathrm{ps}$. The $\mathrm{CW}$ pulse has a peak power of $95 \mathrm{~mW}$ and a width of $0.95 \mathrm{ps}$. The down chirp is $60 \mathrm{GHz}$, but with a plateau near the maximum intensity. The $\mathrm{CCW}$ pulse has less than $5 \%$ of the energy of the $\mathrm{CW}$ pulse. 

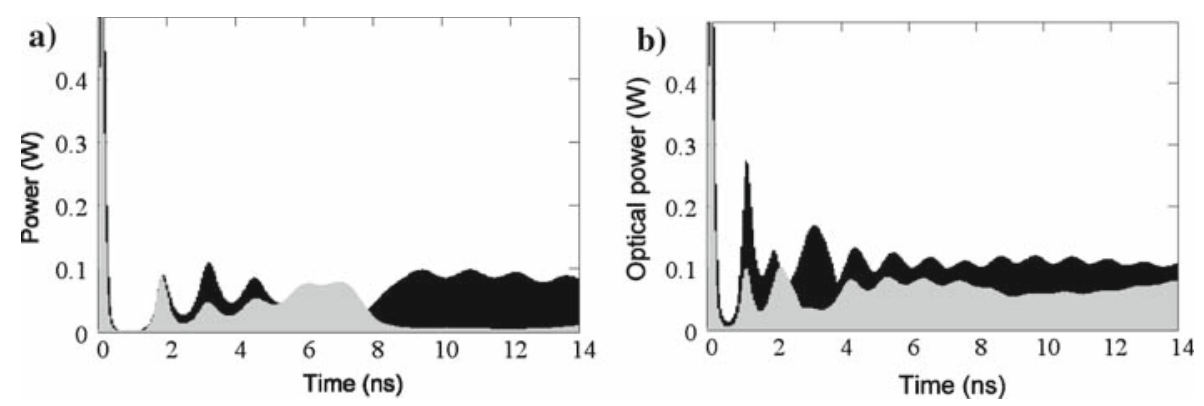

Fig. 17 Simulated output power of the asymmetric $20 \mathrm{GHz}$ RMLL as a function of time. (a) After $8 \mathrm{~ns}$, the laser is quasi unidirectional $(\mathrm{CW}) . \mathrm{I}=60 \mathrm{~mA} \tau_{\mathrm{SA}}=8 \mathrm{ps}$. (b) Stable operation at $\mathrm{I}=67.5 \mathrm{~mA} \tau_{\mathrm{SA}}=5 \mathrm{ps}$
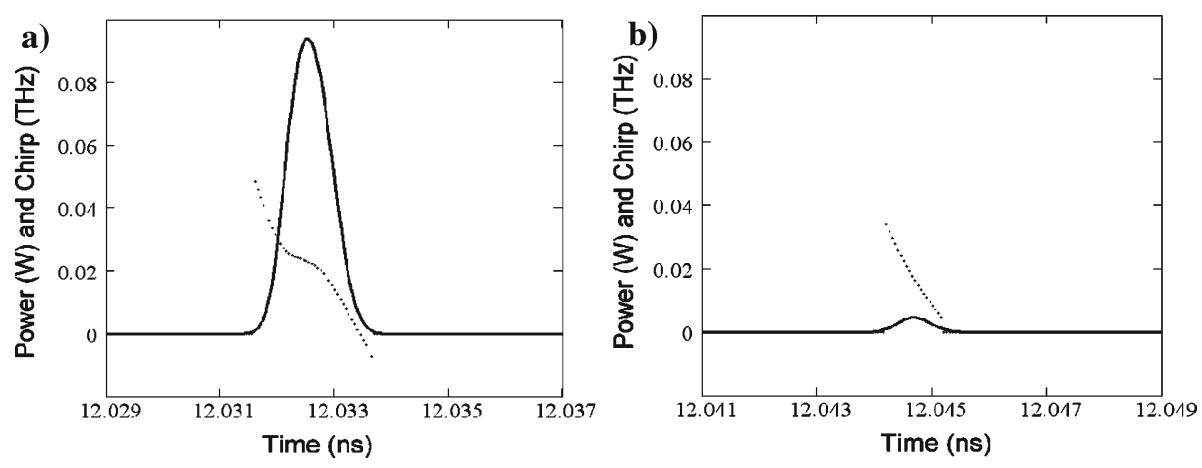

Fig. 18 Simulated CW and CCW output pulses of the asymmetric $20 \mathrm{GHz}$ RMLL when the laser is quasi unidirectional. $\mathrm{I}=60 \mathrm{~mA} \tau_{S A}=8 \mathrm{ps}$ (a) $\mathrm{CW}$ : Peak power $=95 \mathrm{~mW}$, width $=0.95 \mathrm{ps}$, down chirp $=60 \mathrm{GHz}$ (nonlinear) (b) CCW: Peak power $=6 \mathrm{~mW}$, width $=0.75 \mathrm{ps}$, down chirp $=30 \mathrm{GHz}$ ( nearly linear)

For 9 ps $<\tau_{\mathrm{SA}}<13 \mathrm{ps}$, two pulses with different intensities and pulse widths can be supported by the laser. For $\mathrm{I}=67.5 \mathrm{~mA}$ and $\tau_{\mathrm{SA}}=11 \mathrm{ps}$ the $\mathrm{CW}$ pulse is more intense and wider than the $\mathrm{CCW}$ one. The $\mathrm{CW}$ pulse has a peak power of $120 \mathrm{~mW}$, a width of $1.45 \mathrm{ps}$ and a non-linear $50 \mathrm{GHz}$ down chirp. The CCW pulse has a peak power of $45 \mathrm{~mW}$, a width of $0.95 \mathrm{ps}$ and a nearly linear $50 \mathrm{GHz}$ down chirp.

For $\tau_{\mathrm{SA}}>13$ ps the two counter-propagating pulses can have almost the same peak power but the CCW pulse width is slightly narrower. The energy balance between the CW and the $\mathrm{CCW}$ regimes is not really constant. A modulation with a period larger than $4 \mathrm{~ns}$ is commonly observed from the simulation.

A regime which was not perfectly stable in the symmetric regime is however more stable in the asymmetric case. The region is indicated in dark grey areas in Fig. 16, it is for $\tau_{\mathrm{SA}}=5 \mathrm{ps}$ and $65 \mathrm{~mA}<\mathrm{I}<70 \mathrm{~mA}$. At the same settings, the symmetric laser produces pulses heavily modulated in intensity. The simulations of the asymmetric laser show pulses of different intensities and pulse widths with a modulation in peak power $<15 \%$. The $\mathrm{CW}$ and CCW time series over $14 \mathrm{~ns}$ are shown in Fig. 17b. The CW pulse has a peak power of $115 \mathrm{~mW}$, a width of $1.35 \mathrm{ps}$ and a non-linear $50 \mathrm{GHz}$ down chirp. The CCW pulse has a peak power of $65 \mathrm{~mW}$, a width of $0.8 \mathrm{ps}$ and a nearly linear $50 \mathrm{GHz}$ down chirp. 


\section{Conclusion}

A time domain bi-directional model for ring modelocked laser has been presented. The model includes nonlinear effects and a logarithmic gain-carrier relation. The optical bandwidth of the system is controlled by a digital filter. The model has been used to simulate two types of RMLLs (symmetric and asymmetric). Simulation results show that a symmetrical cavity is better for stable modelocking. In that configuration, transitions from the modelocked state of the laser to other operating regimes have been explored with the model. The pulse width increases with the current in the amplifier and decreases with SA carrier lifetime. The $20 \mathrm{GHz}$ design that was simulated contains long passive waveguides, which limits the amount of self phase modulation in the amplifiers, thus the pulses are near transform limited, thus not highly chirped. An asymmetric design has been proposed in order to investigate the possibility of having an integrated RMLL operating in a unidirectional regime. The simulation results of the asymmetric RMLL show that a modelocking regime is more difficult to achieve, however depending on the amount of absorption in the SA in the cavity, the laser can operate with two counter-propagating pulses with significantly different peak intensities.

Acknowledgements The authors would like to thank prof. Daan Lenstra and dr Mirvais Yousefi for stimulating and fruitful discussions on the subject of this paper. This research is supported by the NRC Photonics program, the Towards Freeband Communication Impulse program of the Dutch Ministry of Economic Affairs and COST action 288.

Open Access This article is distributed under the terms of the Creative Commons Attribution Noncommercial License which permits any noncommercial use, distribution, and reproduction in any medium, provided the original author(s) and source are credited.

\section{References}

Avrutin, E.A., Marsh, J.H., Arnold, J.M., Krauss, T.F., Pottinger, H., DeLa Rue, R.M.: Analysis of harmonic (sub)THz passive mode-locking in monolithic compound cavity Fabry-Perot and ring laser diodes. IEE Proc. Optoelectron. 146(1), 55-61 (1999)

Avrutin, E.A., Marsh, J.H., Portnoi, E.L.: Monolithic and multi-GigaHertz mode-locked semiconductor lasers: constructions, experiments, models and applications. IEE Proc.-Optoelectron. 147(4), 251278 (2000)

Bandelow, U., Radziunas, M., Vladimirov, A., Hüttl, B., Kaiser, R.: Harmonic mode-locking in monolithic semiconductor lasers: theory, simulations and experiment. Opt. Quantum Electron. 38, 495512 (2006)

Barbarin, Y., Bente, E.A.J.M., de Vries, T., den Besten, J.H., van Veldhoven, P.J., Sander-Jochem, M.J.H., Smalbrugge, E.J., van Otten, F.W.M., Geluk, E.J., Heck, M.J.R., Leijtens, X.J.M., van der Tol, J.G.M., Karouta, F., Oei, Y.S., Nötzel, R., Smit M.K.: Butt-joint interfaces in InP/InGaAsP waveguides with very low reflectivity and low loss. In: Proc. IEEE/LEOS Benelux Chapter. ISBN 2-960022645, pp. 249-252. Mons, Belgium (2005a)

Barbarin, Y., Bente, E.A.J.M., Heck, M.J.R., Oei, Y.S., Nötzel, R., Smit, M.K.: Passively modelocked 20 and $40 \mathrm{GHz}$ bulk InGaAsP lasers. In: Proc. ECOC 2005, We4P 086, 25-29 September 2005, pp. 1-2. Glasgow, Scotland (2005b)

Barbarin, Y., Bente, E.A.J.M, Mussard, L., Servanton, G., Oei, Y.S., Nötzel, R., Smit, M.K.: Gain measurements of Fabry-Pérot InP/InGaAsP lasers using an ultra high resolution spectrometer. Appl. Opt. 45(34), 9007-9012 (2006)

Barbarin, Y., Bente, E.A.J.M., Heck, M.J.R., Pozo, J., Rorison, J.M., Oei, Y.S., Nötzel, R., Smit, M.K.: 18 GHz Fabry-Pérot integrated extended cavity passively modelocked lasers. In: Proceedings of the European Conference on Integrated Optics, April 25-27, Copenhagen, Denmark (2007)

Bente, E.A.J.M., Smit, M.K.: Ultrafast InP optical integrated circuits. In: Eldada, L.A., Lee, E.-H. (eds.) Photonics West 2006, Optoelectronic integrated circuits VIII, Proceedings of SPIE, vol. 6124, 612419. San Jose, USA (2006) 
Bischoff, S., Mørk, J., Franck, T., Brorson, S.D., Hofmann, M., Fröjdh, K., Prip, L., Sørensen, M.P.: Monolithic colliding pulse mode-locked semiconductor lasers. Quantum Semiclassical Optics. J. Eur. Opt. Soc. B 9(5), 655-674 (1997)

Derickson, D.J., Helkey, R.J., Mar, A., Karin, J.R., Wasserbauer, J.G., Bowers, J.E.: Short pulse generation using multisegment mode-locked—semconductor lasers. IEEE J. Quantum Electron. 28(10), 21862202 (1992)

Hamming, R.W.: Digital filters. Prentice Hall International (UK) Ltd. ISBN: 0-13-212812-6 (1989)

Haus, H.A.: Theory of mode locking with a slow saturable absorber. IEEE J. Quantum Electron. 11(9), 736746 (1975)

Heck, M.J.R., Bente, E.A.J.M., Barbarin, Y., Lenstra, D., Smit, M.K.: Simulation and design of integrated femtosecond passively mode-locked semiconductor ring lasers including integrated passive pulse shaping components. IEEE J. Sel. Top. Quantum Electron. 12(2), 265-276 (2006)

Jury, E.I.: Theory and application of the z-transform method. RE Krieger Pub. Co (1973)

Karin, J.R., Helkey, R.J., Derickson, D.J., Nagarajan, R., Allin, D.S., Bowers, J.E., Thornton, R.L.: Ultrafast dynamics in field-enhanced saturable absorbers. Appl. Phys. Lett. 64(6), 676-678 (1994)

Koumans, R.G.M.P., van Roijen, R.: Theory for passive mode-locking in semiconductor laser structures including the effects of self-phase modulation, dispersion and pulse collisions. IEEE J. Quantum Electron. 32, 478-492 (1996)

Lau, K.Y., Paslaski, J.: Condition for short pulse generation in ultrahigh frequencymode-locking of semiconductor lasers. IEEE Trans. Photon. Technol. Lett. 3(11), 974-916 (1991)

Leegwater, J.A.: Theory of mode-locked semiconductor lasers. IEEE J. Quantum Electron. 32, 17821790 (1996)

Ohno, T., Sato, K., Iga, R., Kondo, Y., Ito, I., Furuta, T., Yoshino, K., Ito, H.: Recovery of 160 GHz optical clock from $160 \mathrm{Gbit} / \mathrm{s}$ data stream using modelocked laser diode. Electron. Lett. 40, 265 (2004)

Olshansky, R., Su, C., Manning, J., Powazinik, W.: Measurement of radiative and nonradiative recombination rates in InGaAsP and AlGaAs light sources. IEEE J. Quantum Electron. 20(8), 838-854 (1984)

Ott, E.: Chaos in dynamical systems. Cambridge Univ. Press. ISBN: 0521811961 (1993)

Radziunas, M.: Numerical bifurcation analysis of the travelling wave model of multisection semiconductor lasers. Phys. D 213, 98-112 (2006)

Takara, H.: High-speed optical time-division-multiplexed signal generation Opt. Quantum Electron. 32, 795 (2001)

Tang, J.M., Shore, K.A.: Amplification of strong picosecond optical pulses in semiconductor optical amplifiers. IEE Proc.-Optoelectron 146, 45-50 (1999)

Van Thourhout, D., Doerr, C.R., Joyner, C.H., Pleumeekers, J.L.: Observation of WDM crosstalk in passive semiconductor waveguides. IEEE Photon. Technol. Lett. 13(5), 457-459 (2001)

Williamson, C.A., Adams, M.J.: Mode locking of a novel split-contact semiconductor laser experiment and theory. IEEE J. Quantum Electron. 40(7), 858-865 (2004)

Williams, K.A., Thompson, M.G., White, I.H.: Long-wavelength monolithic mode-locked diode lasers. New J. Phys. 6, 179 (2004)

Yu, S., Krauss, T.F., Laybourn, P.J.R.: Mode locking in large monolithic semiconductor ring lasers. Opt. Eng. 37, 1164-1168 (1998)

Zhang, L.M., Carroll, J.E.: Large-signal dynamic-model of the DFB laser. IEEE J. Quantum Electron. 28, 604 (1992) 